\title{
The orientation of cognitive maps
}

\author{
MICHAEL PALIJ, MARVIN LEVINE, and TRACEY KAHAN \\ State University of New York at Stony Brook, Stony Brook, New York
}

\begin{abstract}
Given the assumption that the cognitive map is picture-like, then it should have a specific orientation. Can that orientation be determined after a person has learned a path sequentially, that is, by walking through it? Two experiments were conducted to answer this question. In both experiments, subjects were blindfolded and were walked through paths laid out on a floor. In the first experiment, they were required to draw a picture of the path that they had just walked through; in the second experiment, they were required to locate targets in the path under conditions in which their presumed cognitive maps were either aligned or contraligned with the path. All of the subjects in the first experiment drew the first line segment of the path upward, suggesting that this part of the path was fixed in an upward direction in memory. In the second experiment, subjects were more accurate and faster in locating points on the path when the cognitive map was hypothesized to be aligned with the path than when it was hypothesized to be contraligned.
\end{abstract}

Levine, Jankovic, and Palij (1982) had blindfolded people learn a simple path laid out on a laboratory floor. A typical 5-point path is shown in Figure 1. They made the following assumptions: (1) After learning a path, people would represent it as a picture-like image.(2) This image would have some specific orientation with respect to the subject's body. Figure 1 shows a schematic of two subjects, A and B, each with a cognitive map of the indicated path. For both subjects, the cognitive map has the same orientation with respect to his or her body (i.e., in both cases, point 1 is closest to the body, point 2 is "up" or further away, etc.). (3) As with any map, if the cognitive map is aligned with the terrain, then it will be easy to use-stipulated targets will be easy to find; if the cognitive map is badly misaligned, for example, by $180 \mathrm{deg}$ (a condition here called "contraligned"), then it will be difficult to use.

This small theory has a definite implication. If we can specify the orientation of a subject's cognitive map with respect to his or her body, then we can predict whether a given test (to locate a given target point) will be easy or difficult. Suppose in Figure 1 we could specify that the Subjects A and B had the cognitive maps with the orientations indicated. Let us now give each subject (who, remember, is blindfolded) this instruction: "You're at point 4 , and point 5 is directly behind [for Subject A; "in front of" for Subject B] you. Walk in a short-cut to point 1." The assumptions above predict that Subject A would find this test easy and that Subject $B$ would find it difficult.

It would be straightforward to test this prediction if

This research was supported by National Science Foundation Grant BNS78-24716 awarded to Marvin Levine. Requests for reprints should be sent to Marvin Levine, Department of Psychology, State University of New York at Stony Brook, Stony Brook, New York 11794.

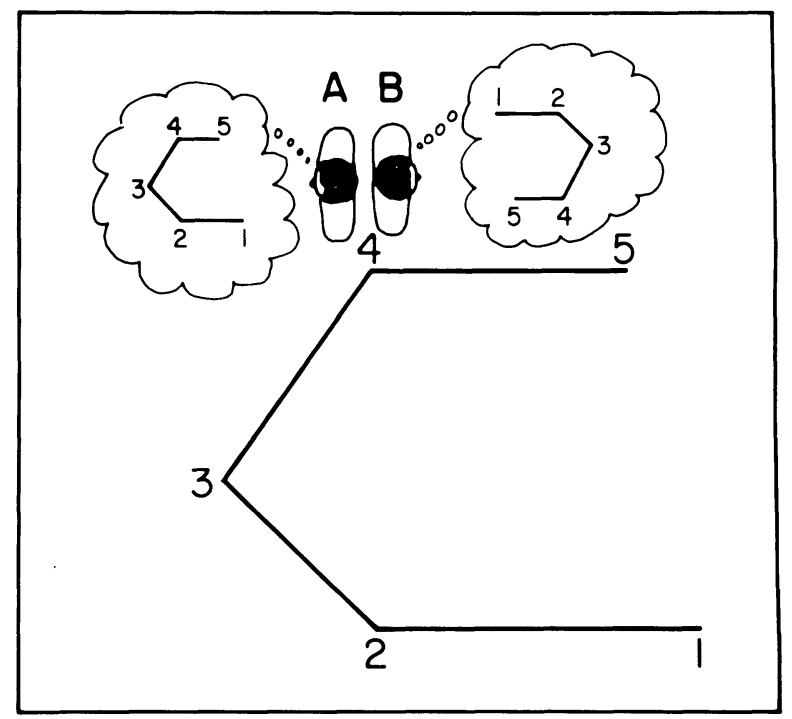

Figure 1. Two subjects standing on the same location on a 5-point path but facing in opposite directions. Subject A's cognitive map is aligned with the path; Subject B's is not.

we could specify the orientation of a subject's cognitive map. Levine et al (1982) used a method of teaching the path that gave face validity to this specification. The subject learned the path by viewing a map of that path: The subject lifted the blindfold and studied for $20 \mathrm{sec}$ a picture of the path. This picture was held rigid for the $20 \mathrm{sec}$, in a fixed orientation. Levine et al. assumed that after the subject again donned the blindfold, he or she would have a memory image of the map just seen and that this cognitive map would have the same orientation in relation to the subject's body as the originally seen map. Thus, Levine et al. specified the orientation of the cognitive map and carried out the test of the prediction 
embodied in Figure 1. The results were decisive: When the presumed cognitive map in the presumed orientation was aligned (cf. A in Figure 1), subjects virtually always walked toward (angle error $<90 \mathrm{deg}$ ) the target; when the map was contraligned ( $B$ in Figure 1), subjects walked away from the target (angle error $\geqslant 90 \mathrm{deg}$ ) on about one-third of the tests. This is the aligned-contraligned (A-C) effect.

In a second experiment, the subjects learned the path slightly differently. Instead of having the subject lift the blindfold and look at the map, the experimenter took the subject's index finger and traced it over the path on the map, moving the finger in the sequence from point 1 to point 5 . In this case, the subject's cognitive map was not a memory image of a visual display but was constructed from the finger tracing. Nevertheless, Levine et al. (1982) assumed that this constructed cognitive map would have the same orientation in relation to the subject's body as the original, explored map. For this experiment also, then, they were able to test the prediction from the theory. Again, they found the A-C effect, that the subjects were clearly and significantly worse on the contraligned tests.

In both the above experiments, the subjects learned the path by experiencing a map of that path. In both cases, it was plausible to assume that the cognitive map had the same orientation with respect to the subject's body as the originally experienced map. However, a third way of learning the path is possible: the subject may be walked through the path directly. According to Levine et al. (1982), this method also yields a picturelike representation. If one could specify the orientation of the resulting image, one should be able to carry out the A-C test. The difficulty is in specifying the orientation. There is no obvious possibility comparable to the map condition. Nevertheless, we saw a plausible basis for hypothesizing about the orientation of the presumed cognitive map following learning by waiking.

Levine (1982), in an analysis of you-are-here maps, proposed that, when people view a fixed vertical map, they tend to interpret the upper part of the map as referring to the terrain that was forward, that is, ahead of them. He called this the forward-up-equivalence and showed that, under certain conditions, this tendency caused people to become lost (see Levine, Marchon, \& Hanley, in press). If people tend to equate forward movement in a terrain with verticality in a map, then that provides a clue to how subjects walking through a path might form an image of that path. Specifically, we assumed that as a subject walks forward from point 1 to point 2 , he or she imagines point 2 as above (or, perhaps, forward of) point 1 . This fixes the first segment of the cognitive map in relation to the subject's body. We further assumed that the image of the rest of the path would follow from the orientation of that initial segment. In other words, we assumed the following corollary to the forward-up equivalence: When a person walks through a simple path, he or she codes the 1-to-2 seg- ment as "up"; the rest of the path is coded in relation to that segment. The cognitive maps of both subjects in Figure 1 manifest such coding. Thus, for subjects who learn the path by walking, this corollary, that 1-to-2 is up, specifies the orientation of the cognitive map in relation to their bodies. It, therefore, permits the same tests of the theory as those performed by Levine et al. (1982).

To evaluate this theory, two experiments were performed. The first experiment was an attempt at direct verification of the corollary. The subjects learned simple paths by being walked through them and were required to draw a picture of the path. If the corollary is correct, the path would be expected to have been drawn with segment 1.2 vertical. In the second experiment, the subjects again learned simple paths by walking through them but were then given tests that were aligned or contraligned, as defined by the corollary. If the walkgenerated cognitive map had the orientation specified by the corollary, then the A-C effect would be expected to appear.

\section{EXPERIMENT 1}

This experiment is similar to the third experiment of Levine et al. (1982), in which subjects learned 5-point paths by being walked through them while blindfolded. That experiment suggested that subjects had developed cognitive maps with the demonstration that they could take shortcuts between nonadjacent points. The present experiment was different only in that the subjects were required to draw a picture of their image of the path.

\section{Method}

Subjects. Eight undergraduates from the State University of New York at Stony Brook were paid for their participation in this experiment.

Stimuli. Four 5-point paths similar to the path shown in Figure 1 were used. The paths were made with tape and were laid out on the floor of a large empty room (approximately $10 \times 15 \mathrm{~m}$ ). The lengths of the line segments on the paths varied from 80 to $350 \mathrm{~cm}$. On two of the paths the subject had to turn to the left when going from point 2 to point 3 , whereas on the remaining two paths the subject had to turn to the right. The degree of turning varied on ail of the paths, with only one path having a right-angle turn at point 2 . On all of the paths, the line segment 4-5 ran parallel to the line segment 1-2 (cf. Figure 1).

Additional materials included a blindfold, a chair with wheels, a standard stopwatch, 10 cartoons taken from popular magazines, an ink marker, and a square pad of white paper $(457 \times 457 \mathrm{~mm})$.

Procedure. The subjects were run individually in a single session lasting less than $1 \mathrm{~h}$. At the beginning of the session, the subject was seated in a wheelchair outside the room that contained the paths. The subject was told that he/she would be blindfolded and would learn a series of 5-point paths by being walked through them three times each by the experimenter. The walk would always begin at point 1 and would end at point 5 . A drawing of a sample path was shown to the subject, and the experimenter provided a narrative of the learning experience. It is important to note that the drawing of this sample had the line segment 1-2 horizontal, going from left to right. Also, unlike the paths that the subject would be walked through, the 
sample path did not have the line segment 4-5 running parallel to line segment $1-2$.

After having given the narrative about walking through the path, the experimenter described the test to the subject. The subject was told that at test time he/she would be wheeled to a test point and would be asked to stand. The subject would then be told which point on the path he/she was standing on and which direction relative to another point on the path he/she was facing. The subject would then be told a target point on the path to which he/she should walk. For example, the experimenter might have said, "You are standing on point 4 , and point 5 is directly in front of you; walk to point $3 . "$

After these instructions, the subject was blindfolded and wheeled into the room with the paths. The subject was then run through two practice problems to familiarize him/her with the procedure. The first practice path was only a 4-point path and was used to accustom the subject to walking around while blindfolded. After three walks through the first practice path, the subject was tested on the path. On the second practice path, a 5-point path, the subject was walked through the path three times and was then wheeled away from the path. The subject was taken to another part of the room and was asked to remove the blindfold. The subject was now told that there was another task for him/her to do, which consisted of rating two cartoons for their humorousness. The subject rated the cartoons separately on a scale from 1 to $10(1=$ not funny, $10=$ very funny $)$. After having rated the cartoons, the subject was asked to recall the path he/she had just walked through and to give a rating of his/her memory of the path on a 10-point rating scale $(1=$ no memory, $10=$ excellent memory of the path). After having given the memory rating, the subject was handed a square white pad of paper and an ink marker and was asked to draw a picture of the path as it appeared in his/her mind. After having drawn the picture, the subject replaced the blindfold and was wheeled back to the path and tested on it.

After the test of the second practice path, the subject was told that the main part of the experiment was about to begin. All of the experimental problems would be identical in format to the procedure used in the second practice problem. The subject was encouraged to do his/her best in both the learning and the testing phase. Any questions the subject may have had were answered. The subject was then wheeled to the first experimental path. After four experimental problems, the subject was interviewed about his/her experience in the experiment, debriefed, paid, and excused from the experiment.

\section{Results and Discussion}

During the experiment, various measures of the subjects' performance on the tests of the paths were taken. This was done primarily to make sure that the procedure used here followed that of Levine et al. (1982). The datum of interest, of course, comes from the drawings made by the subjects. The question is whether the corollary is confirmed, that is, whether the subjects drew the path with segment 1-2 up. The answer is clear and straightforward: All subjects drew the line segment 1.2 upward on the pad of paper. That is, all of the subjects tended to start the drawing of the path near the bottom of the pad and then drew a line that was perpendicular to the bottom of the pad. All 32 drawings generated by the subjects had the line segment 1.2 within $30 \mathrm{deg}$ of the perpendicular. In fact, all but three of these drawings were within 5 deg of the perpendicular. These results strongly support the corollary that a walk-generated cognitive map has a standard orientation in memory, with the first line segment of the path fixed in an upward direction. Thus, the forward-up equivalence appears to be operating when the subject is constructing the cognitive map. In the interview after the experiment, the subjects said that they had made an image and that the image looked like their drawing of the path.

It could be argued, however, that the subjects were not relying upon their mental images when asked to draw a picture of the path and, instead, came to the experiment with a bias to draw any numbered path with 1-2 going upward. That is, their experience of walking through a path is not relevant when it comes to drawing a picture of the path. When asked, a person may always draw a path with the first segment vertical and up. To counter this objection, a small, informal study was conducted. Students in several undergraduate classes at Stony Brook were asked to imagine and draw a picture of a 5-point path. As an example, the students were shown the picture of the sample path shown to subjects in the current experiment (recall that in this picture the line segment 1-2 was horizontal, going from left to right). The students were given square pieces of white paper to draw the paths on. A total of 95 drawings were obtained. It was decided to divide the drawings into four categories reflecting the direction in which the line segment 1-2 was drawn: upward, left to right, downward, and right to left. A chi-square analysis of the frequencies in these categories indicates that there was no preferred direction in which 1-2 was drawn. The category with the greatest frequency, $41 \%$, was left to right, which was the direction of 1.2 on the example path. Thus, the result of the main experiment was not due to any general bias in drawing paths.

In summary, people appear to fix the orientation of the cognitive map on the basis of their learning experience with the path. The corollary that people translate their first forward movement on the path into an upward line segment in their cognitive map is confirmed. In other words, people apply the forward-up equivalence in constructing the cognitive map and thereby standardize its orientation.

\section{EXPERIMENT 2}

In this experiment, we attempted to validate the theory in a more general way, to substantiate the claim that a walk-generated cognitive map is pictorial and has a specific orientation. By assuming that the 1-2 segment was always up, we could present people with aligned and contraligned tests.

\section{Method}

Subjects. Sixteen subjects were recruited from the undergraduate population at the State University of New York at Stony Brook. None of these had served in the previous experiment. Eight of these subjects were males, and eight were females. The subjects were paid for their participation.

Stimuli. Eight 5-point paths were used. Four of these paths had been used in the previous experiment, and four new paths with similar properties were constructed (e.g., line segment 4-5 was parallel to line segment 1-2). The experiment was conducted 
in the same room that had been used previously. Additional materials included 24 cartoons taken from popular magazines, a stopwatch, a protractor with a long thin wire joined at the center of its straightedge, and a 2-m-long wooden staff.

Design and Procedure. Each subject participated in two 45-min sessions that were separated by 1 or 2 days. The general procedure used in this experiment was very similar to that used in the previous experiment. At the beginning of the first experimental session, the subjects were seated in the wheelchair outside the room that contained the paths. They were instructed about the procedure and then blindfolded and wheeled into the room. Again, the subjects were given two practice paths that followed the format of the practice problems of the previous experiment, except that the subjects were not asked to draw a picture of the path before the test. At test time, the subject was told which point he/she was standing on and in which direction he/ she was facing. The subject was then told the target point. Just before the subject was told his/her location on the path, he/she was given a long wooden staff to hold slightly off the ground. After the subject had walked to the target point, he/she was to let the bottom of the stick touch the ground to indicate the location of the target point on the floor. A large protractor was then used to measure the angle deviation between the true target point and where the subject had placed the point of the stick.

The two practice problems were followed by four experimental problems which completed the first session. At the beginning of the second session, the procedure was reviewed for the subjects and they were given four more experimental problems. At the end of the second session, the subjects were interviewed.

Of the eight tests, four were specified, a priori, as being aligned, and four as being contraligned. If the subjects fixed the orientation of the cognitive map by having the line segment 1-2 going upward, then the specification of aligned and contraligned trials is straightforward. This can be seen by referring to Figure 1. If, for the cognitive map of that figure, line segment 1-2 is pointing upward, then point 5 will be below point 4 . Now, if a subject is placed on point 4 with point 5 directly behind him/her (cf. Subject A in Figure 1), then that subject will be having an aligned test. But if the subject is standing on point 4 facing point 5 (cf. Subject B in Figure 1), then he/she will be having a contraligned test. Of the eight tests that each subject received, four were aligned and four were contraligned.

\section{Results and Discussion}

Two measures of performance were made at test time: (1) error between the true target point and the target point location indicated by the subject, and (2) the amount of time taken by the subject to indicate the target point. Our hypothesis is that performance should be more accurate and faster under the aligned test condition than under the contraligned test condition.

Both of these measures were analyzed by a repeatedmeasures analysis of variance. The results show that the aligned-condition performance was significantly more accurate (mean $=46 \mathrm{deg}$ ) than the contraligned-condition performance $($ mean $=69 \mathrm{deg})[\mathrm{F}(1,14)=4.64, \mathrm{p}<$ $.05]$. Also, performance under the aligned condition was significantly faster (mean $=25 \mathrm{sec}$ ) than that under the contraligned condition (mean $=34 \mathrm{sec})[\mathrm{F}(1,14)=6.04$, $\mathrm{p}<.05]$. This is the A-C effect. It confirms our assumptions that the subjects' walk-generated cognitive maps were pictorial and had a specific orientation in memory fixed by the upward representation of the first forward movement on the path.
During the postexperiment interview, most subjects reported that they had made mental images of the paths that they had learned. They typically said that they had imagined the path as a drawing on a vertical pad or blackboard. A few of the subjects, however, said that they had imagined the path on the floor. They had pictured it, that is, not as a vertical cognitive map, but as a scene, almost like seeing the paths on the floor around them through the blindfold. Not enough of these subjects could be identified to meaningfully distinguish their results from those of the subjects who claimed to have used a cognitive map representation. Further research is needed to clarify what subjects mean when they say that they have imagined the path on the floor. Also, what role orientation plays in this type of mental representation is not immediately clear.

These experiments extended the findings of Levine et al. (1982). The earlier results had supported the assumption that the cognitive map of adult subjects is picture-like. Such an assumption implies that the cogni. tive map, at any moment in time, has a specific orientation. This characteristic, in turn, implies that certain tests (aligned) should be easy and others (contraligned) relatively difficult. The previous research confirmed this A-C effect, but only for cognitive maps derived from the study of maps of the space, not from exploration of the space itself.

The present research was concerned with the latter condition, with showing the A-C effect for cognitive maps constructed from direct exploration of the space. In order to specify alignment, we conjectured that the first (1-2) segment of the path would be represented as going up in the cognitive map and that this would anchor the remainder of the map. Experiment 1 provided some confirmation of this conjecture by showing that subjects drew the paths with the 1-2 segment up. Experiment 2 demonstrated the A-C effect. As Levine et al. (1982) argued, this finding not only supports the assumption of a picture-like representation of the space, but is not derivable from any sequential (e.g., trigonometric) description of the space. That is, it is not derivable unless some arbitrary ad hoc assumptions are added. From the assumption of a picture-like representation, on the other hand, the A-C effect is a direct consequence.

\section{REFERENCES}

Levine, M. (1982). You-are-here-maps: Psychological considerations. Environment and Behavior, 14, 221-237.

Levine, M., Jankovic, I. N., \& PaliJ, M. (1982). Principles of spatial problem solving. Journal of Experimental Psychology: General, 111, 157-175.

Levine, M., Marchon, I. O., \& Hanley, G. (in press, 1984) The placement and misplacement of you-are-here maps. Environment and Behavior.

(Manuscript received for publication November 29, 1983.) 